\title{
GAS AND DUST IN ULTRALUMINOUS GALACTIC NUCLEI
}

\author{
N.Z. SCOVILLE \\ California Institute of Technology \\ Astronomy 105-24, Pasadena, CA 91125 \\ AND \\ M.S. YUN \\ National Radio Astronomy Observatory \\ P. O. Box 0, Socorro, NM 87801
}

\begin{abstract}
Millimeter-wave interferometry has clearly shown the existence of enormous masses $\left(10^{9}-10^{10} M_{\odot}\right)$ of molecular gas concentrated in the nuclear regions $(R<500 \mathrm{pc})$ of many luminous and ultraluminous infrared galaxies. In these systems, molecular gas is an obvious source of fuel for nuclear starbursts and active galactic nuclei (AGN). In several ultraluminous systems (e.g., Arp 220 and Mrk 231), there now exists CO (2-1) interferometry at $\leq 1$ " resolution which reveals for the first time extremely dense, gaseous accretion disks on the scale 50-300 pc. Based on the low velocity dispersion of the molecular gas in the nuclear disks, we believe them to be extremely thin $(10-50 \mathrm{pc})$. In addition, high brightness temperatures in the CO lines $(10-20 \mathrm{~K})$ imply that these disks are nearly uniformly filled with a continuous gas distribution, rather than being relatively isolated, self-gravitating GMCs. Although the gas is 'uniformly' distributed, the gas densities must be high, $>10^{4} \mathrm{~cm}^{-3}$. When viewed near the plane of the disk, the central AGNs, if they exist, will be totally obscured at optical and near infrared wavelengths. In Mrk 231, our line of sight is probably within $60^{\circ}$ of the disk axis, but in Arp 220 the disk is closer to edge-on. In fact, recent near infrared imaging of Arp 220 with the NICMOS camera on the $H S T$ reveals totally opaque dust disks embedded within the central star clusters of both nuclei.
\end{abstract}




\section{Introduction}

The critical role played by dense molecular gas in the activity of galactic nuclei has been appreciated only in the last decade. This gas, which is virtually undetectable via $21 \mathrm{~cm} \mathrm{HI}$ observations (except in rare cases of absorption), has now been studied at millimeter wavelengths by both single-dishes and aperture synthesis, the latter achieving $<1$ " resolution in recent work. In the more luminous systems, the large masses of molecular gas are undoubtedly responsible for the prodigious starburst activity and very likely responsible for feeding accretion into central, pc-scale AGN accretion disks. The gas is important not only because it can form stars; it is also extremely dissipative and efficient in radiating bulk rotational energy and transferring angular momentum to larger radii. Despite its energetic environment, the molecular gas probably remains at temperatures of less than $100 \mathrm{~K}$ due to extremely effective cooling in molecular lines and the associated dust continuum. The bulk of the far-infrared luminosity can be characterized by color temperatures $40-80 \mathrm{~K}$ and the masses of dust derived from the far-infrared opacity are consistent with the molecular gas masses assuming reasonably standard gas-to-dust abundance ratios (100-500 by mass). Here we describe in detail the recent results on Arp 220 and Mrk $231 \mathrm{which}$, to some extent, represent earlier and later evolutionary phases of the ultraluminous galaxy phenomena.

\section{Arp 220}

Arp 220 is the prototypical, ultraluminous infrared galaxy with a luminosity at $8-1000 \mu \mathrm{m}$ of $1.5 \times 10^{12} L_{\odot}$, clearly placing it in the luminosity regime of quasars. In the visual, Arp 220 exhibits two faint tails which are probably the result of a past tidal interaction (cf Joseph \& Wright 1985) and in the nucleus, high resolution near infrared and radio imaging reveals a double nucleus with spatial separation $0.95^{\prime \prime}$ (Graham et al 1990; Norris 1988). The projected separation of the nuclei corresponds to $330 \mathrm{pc}$; this double nucleus structure, together with the extended optical tails, suggests that the galaxy is in the final stage of galactic merging.

Single-dish observations of the CO in Arp 220 have revealed an extraordinarily high $\mathrm{CO}$ luminosity, corresponding to an $\mathrm{H}_{2}$ mass of $2 \times 10^{10} M_{\odot}$ assuming a Galactic conversion ratio (Sanders et al. 1991), and $3 \mathrm{~mm}$ aperture synthesis has revealed that the bulk of this $\mathrm{CO}$ luminosity originates from the central kpc (Scoville et al. 1991). Recently, this system has been mapped in CO (2-1) at $1^{\prime \prime}$ resolution (Scoville et al. 1997; Downes 1998). This new work reveals for the first time multiple components in the dense gas: peaks corresponding to each of the double nuclei (separated by $0.95^{\prime \prime}$ at $P A=101^{\circ}$ ), and a more extended disk-like structure at $P A=53^{\circ}$ sim- 

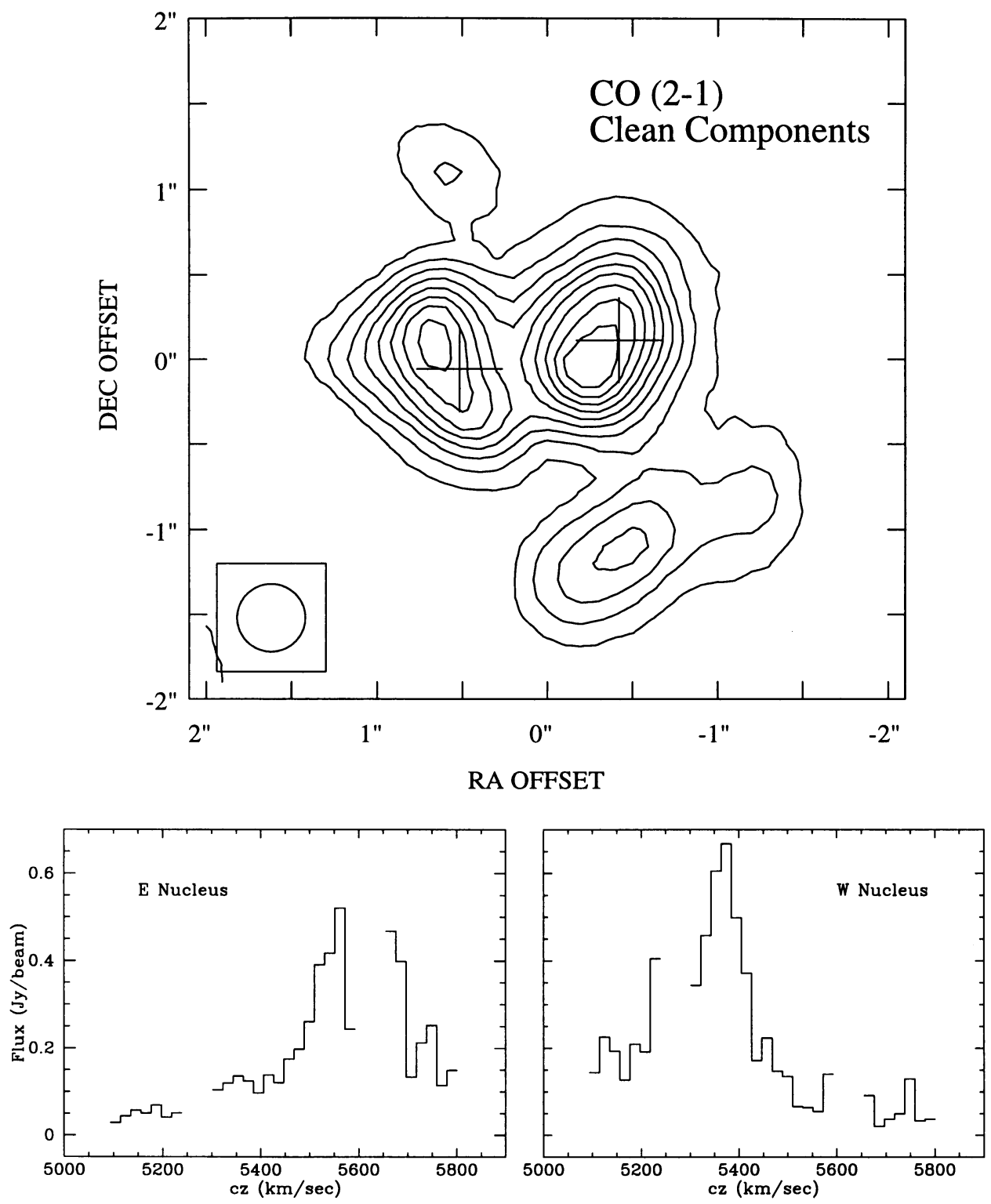

Figure 1. The integrated intensity of $\mathrm{CO}(2-1)$ emission from the brightest 'clean' components (Scoville et al. 1997b) is shown with the crosses indicating the positions of the two radio nuclei (Baan \& Haschick 1992). Spectra of the clean components at the two nuclei are shown at the bottom. Their double horned shapes suggest gas disks within each of the nuclei. 
ilar to the dust lane seen in optical images. Approximately two-thirds of the total $\mathrm{CO}$ emission (and presumably the $\mathrm{H}_{2}$ mass) coincides with the compact double nucleus region.

Detailed modeling of the CO line profiles in Arp 220 using a Doppler image-deconvolution technique similar to that used previously for NGC 1068 (Scoville et al. 1983) yields a best-fit CO emissivity distribution and rotation curve which are mutually consistent - in the sense that, if the total mass distribution follows the $\mathrm{CO}$ emissivity, it produces the derived rotation curve. The implied CO-to- $\mathrm{H}_{2}$ conversion ratio is 0.45 times the Galactic value if the bulk of the mass resides in the molecular gas rather than the stars. The total molecular gas mass for Arp 220 is then inferred to be approximately $\sim 9 \times 10^{9} M_{\odot}$. An important result of the line profile modeling is that the intrinsic velocity dispersion in the extended disk is only $90 \mathrm{~km} \mathrm{~s}^{-1}$. Assuming that the disk gas is entirely self-gravitating, its thickness (FWHM) is only 16 pc. The mean density in this disk is then $2 \times 10^{4} \mathrm{~cm}^{-3}$, a value which is entirely consistent with the strong emission from high dipole moment molecules such as $\mathrm{HCN}$ and $\mathrm{HCO}^{+}$(cf Solomon et al. 1992). If the gaseous disk is only partially self-gravitating (i.e., the potential is dominated by a stellar disk), then the gas thickness can be a few times larger.

From the high brightness temperatures of the observed CO emission $(17-21 \mathrm{~K})$ and comparison with the infrared color temperature, it is clear that the area filling factor of the disk is very high $(\sim 0.25)$, and therefore that the gas must fairly uniformly fill the disks rather than exist in discrete, self-gravitating clouds. This represents a major change in our picture of the central gas distributions in merging galaxies: even in these highly disturbed systems, the gas has relaxed to a rotationally supported disk which, due to its extremely high surface density and relatively low velocity dispersion, must be very thin. Presumably, this rapid relaxation has occurred as a result of the very strong dissipation in the interstellar gas. Direct observational evidence for the existence of a thin disk in the center of Arp 220 has recently been provided by high resolution near-infrared imaging obtained with the NICMOS camera on the HST (Scoville et al. 1998). The near-infrared images clearly show the dominant western nucleus to be crescent-shaped, as though the central star cluster has been partially obscured by an embedded, opaque dust disk.

The fact that the locations and velocities of the nuclei are mutually consistent with their being situated in the molecular disk is suggestive that the nuclei are indeed orbiting with the disk plane. If they were above or below the disk and simply seen in projection against the disk, the positions and velocities would be unlikely to match those of the gas. The results are consistent with a geometry for the central region of Arp 220 in which 


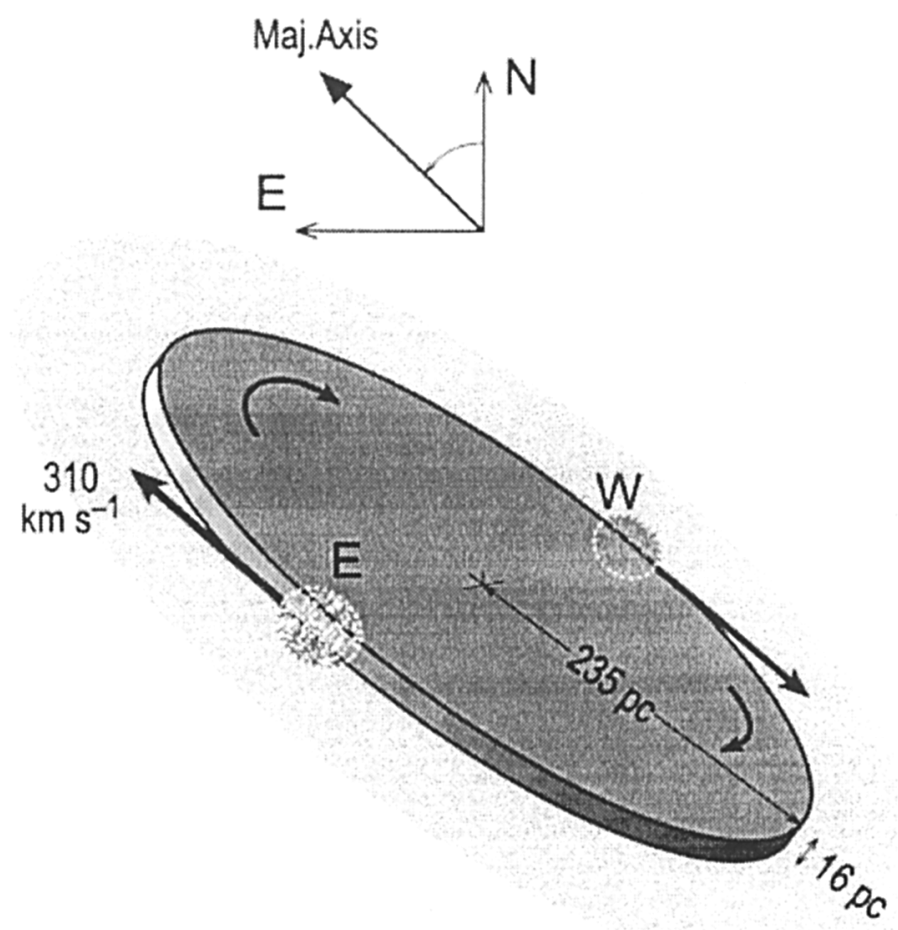

Figure 2. Schematic of the nucleus of Arp 220 showing the central molecular gas disk with the double infrared/radio nuclei orbiting at the outer edge of the nuclear gas disk. Based on the elongation of the molecular disk, the adopted major axis is at $P A=45^{\circ}$, and the disk is inclined at approximately $40^{\circ}$ to the line of sight. The infrared nuclei then lie along a line between the major and minor axes of the gas disk $\left(69^{\circ}\right.$ from the major axis in the disk plane). Approximately two-thirds of the total molecular emission arises from this disk, the remaining from a more extended disk with approximately 10 times lower surface density.

both the molecular gas and the double nuclei are situated in an inclined, rotationally supported disk. This geometry is shown schematically in Figure 2.

\section{Mrk 231}

At a distance of $174 \mathrm{Mpc}$, Mrk 231 is one of the most luminous objects in the local universe, with an infrared luminosity of $3.5 \times 10^{12} L_{\odot}$ (Soifer et al. 


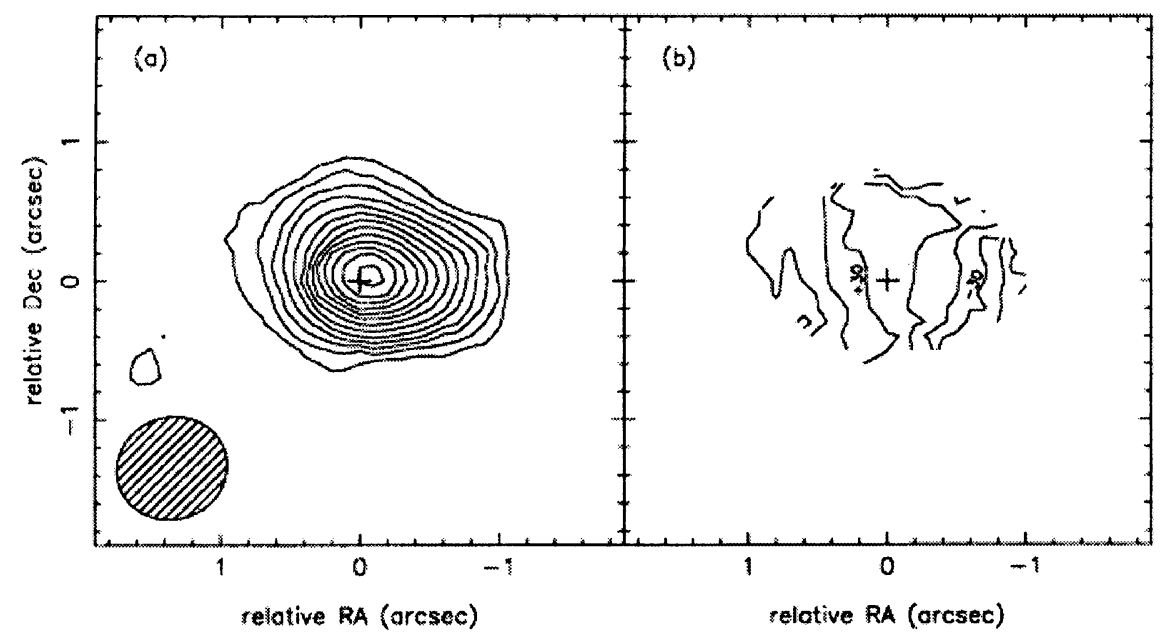

Figure 3. Velocity integrated flux density map (left) and mean velocity map (right) from the CO (2-1) observations of Mrk 231 at $1^{\prime \prime}$ resolution (Bryant \& Scoville 1996).

1989). The optical morphology indicates two linear, low surface brightness features that may be tidal tails (Hutchings \& Neff 1987, Sanders et al. 1987). The optical spectrum of the nucleus reveals Seyfert I emission line features and a highly reddened continuum and several systems of low ionization absorption lines at velocities up to $8240 \mathrm{~km} \mathrm{~s}^{-1}$ relative to the systemic velocity (Boksenberg et al. 1977, Boroson et al. 1991).

Recently, Bryant \& Scoville (1997) mapped the CO (2-1) emission at $0.8^{\prime \prime}$ resolution. The source is well resolved with a deconvolved size of 0.3 $\times 1.0^{\prime \prime}$ (Figure 3). The major axis radius corresponds to $420 \mathrm{pc}$. A velocity gradient is also seen in the CO (2-1) line and the kinematic major axis agrees in position angle with the $\mathrm{CO}$ isophotal major axis, suggesting as in Arp 220 that the molecular emission arises from an inclined disk. The peak beam-diluted brightness temperature of the CO (2-1) line is $14 \mathrm{~K}$, indicating that the molecular gas also has a high area filling-factor.

Two factors suggest that the disk in the nucleus of Mrk 231 is more nearly pole on: the low visual extinction $\left(A_{\mathrm{V}}=2 \mathrm{mag}\right.$, Boksenberg et al. 1977) derived from the optical spectrum and the narrow CO line width (189 $\mathrm{km} \mathrm{s}^{-1}$, Bryant \& Scoville 1997). The near pole-on geometry is further supported by the detection of the same molecular disk in the $21 \mathrm{~cm}$ $\mathrm{HI}$ absorption but the absence of any absorption towards the central radio source in a VLBA experiment by Carilli et al. (1998). A useful lower limit 
to the molecular gas mass was derived by Bryant \& Scoville (1997) based on the assumption that the CO line was optically thin and that the excitation temperature is such as to give the minimum gas mass consistent with the observed brightness temperature. This lower limit on the mass is 1.3 $\times 10^{9} M_{\odot}$, assuming a standard Galactic abundance of $\mathrm{CO}$ relative to $\mathrm{H}_{2}$. This lower limit to the mass is only a factor of 5-10 less than that derived under the standard assumptions of optically thick CO line emission.

\section{Concluding Remarks}

Several systems imaged at high spatial resolution in the molecular gas (e.g., VV114 and NGC 6240) bear striking similarities to Arp 220 and Mrk 231 (Yun et al. 1994, Bryant \& Scoville 1997). Several exhibit CO peaks between double infrared/radio nuclei, clear rotational velocity gradients suggestive of a centrifugally supported disk, and relatively strong high-density molecular line tracers.

These dense molecular-gas accretion disks may play a critical role in the evolution of ultraluminous galaxy nuclei and in their energy release. The dense gas will undoubtedly promote extremely high rates of massive star formation and the disks are also efficient in removing angular momentum - thus feeding and building up a central active nucleus at rates up to 100 $M_{\odot} \mathrm{yr}^{-1}$. This latter process is probably most important in the most evolved and highest luminosity systems such as Mrk 231.

The characteristics of the molecular gas in the nuclear disk of Arp 220 are extraordinarily different from those in the centers of nearby lower luminosity galaxies. In the Galactic center, the molecular gas $\left(\leq 10^{8} M_{\odot}\right)$ is mostly contained in very massive, self-gravitating, molecular clouds (e.g., Sgr B2 or the Sgr A complexes) with relatively little intercloud molecular gas and a half-thickness for the cloud distribution of 60-120 pc. In Arp 220, the total gas mass is a factor $\geq 100$ larger within a comparable radius, and it appears inescapable that this gas is much more uniformly distributed (i.e., less cloud-like) and confined to an extremely thin disk (16 pc halfthickness). Uniformity of the gas in this disk is suggested by the high area filling factor $(\simeq 0.25)$ of the molecular gas derived from the observed $\mathrm{CO}$ line brightness temperatures. And given the high area filling factor and small scale height, the mean free path of clouds within this layer should be only a few disk scale heights. If we conservatively estimate the cloud-cloud collisional mean free path as $\leq 50 \mathrm{pc}$, then the mean collision time is only $5 \times 10^{5}$ yr. With such short cloud-cloud collision times and high velocities, any preexisting clouds would be rapidly disrupted and their matter spread uniformly in the disk.

The two galaxies discussed here (Arp 220 and Mrk 231) may repre- 
sent two critical phases in the merging galactic nuclei scenario which has been suggested to link the ultraluminous infrared galaxies with AGNs and quasars. Arp 220 is still very much dust-enshrouded but shows two distinct nuclei, whereas the latter has only one major nucleus and optical spectroscopy shows both the broad emission lines characteristic of a Seyfert 1 nucleus and broad absorption lines. In the context of the ultraluminous IR galaxy/quasar evolutionary models (e.g., Sanders et al. 1988), Arp 220 would represent an earlier phase just prior to the merging of the two galactic nuclei, and Mrk 231 the phase in which the quasar-like nucleus has been blown free of dust, at least above and below the central nuclear gas disk.

\section{Acknowledgments}

This research is supported in part by NSF Grant AST96-13717 and the Norris Planetary Origins Project. We thank Nanci Candelin for help in preparation of this manuscript.

\section{References}

Boksenberg, A., Carswell, R.F., Allen, D.A., Fosbury, R.A.E., Penston, M.V., \& Sargent, W.L.W. 1977, MNRAS, 178, 451

Boroson, T.A., Meyers, K.A., Morris, S.L., \& Persson, S.E. 1991, Ap.J., 370, L19

Bryant, P.M. 1996, Ph.D. thesis, California Institute of Technology

Carilli, C.L., Wrobel, J.M., \& Ulvestad, J.S. 1998, Ap.J., in press

Downes, D. 1998, IAU Symposium 184, in press

Graham, J.R., Carico, D.P., Matthews, K., Neugebauer, G., Soifer, B.T., \& Wilson, T.D. 1990, Ap.J. (Letters), 354, L5

Hutchings, J.B. \& Neff, S.G. 1987, Ap.J, 92, 14

Joseph, R.D. \& Wright, G.S. 1985, MNRAS, 452, 599

Larkin, J.E., Armus, L., Knop, R.1A., Matthews, K., \& Soifer, B.T. 1995, Ap.J., 452, 599

Norris, R.P. 1988, MNRAS, 230, 345

Sanders, D.B., Scoville, N.Z., \& Soifer, B.T. 1991, Ap.J., 370, 158

Sanders, D.B., Soifer, B.T., Elias, J.H., Madore, B.F., Matthews, K., Neugebauer, G., \& Scoville, N.Z. 1988, Ap.J., 325, 74

Scoville, N.Z., Evans, A.S., Dinshaw, N., Thompson, R., Rieke, M., Schneider, G., Low, F.J., Hines, D., Stobie, B., Backlin, E., \& Epps, H. 1998, Ap.J. (Letters), January 15,1998 issue

Scoville, N.Z., Young, J.S., \& Lucy, L.B., 1983, Ap.J., 270, 443

Solomon, P.M., Downes, D., \& Radford, S.J.E. 1992, Ap.J. (Letters), 387, L55

Yun, M.S., Scoville, N.Z., \& Knop, R.A. 1994, Ap.J. (Letters), 430, L109 